\title{
A Practical Measurement Method of the Occupied Bandwidth for 8-VSB DTV Signal Using Modified ACPR
}

\author{
Young Soo Kim ${ }^{1 *}$, Bong Gyou Lee ${ }^{2}$ and Kyeongmin Song ${ }^{3}$ \\ ${ }^{1}$ College of Electronic and Information, Kyung Hee University \\ Yongin, Gyoungkido, Korea \\ [e-mail: yskim@khu.ac.kr] \\ ${ }^{2}$ Graduate School of Information, Yonsei University \\ Seoul, Korea \\ [e-mail: bglee@yonsei,ac,kr] \\ ${ }^{3}$ Wibtel Co., LTD \\ Seongnam, Gyoungkido, Korea \\ [e-mail: kmsong@wibtel.com] \\ *Corresponding author: Young Soo Kim
}

Received March 12, 2018; revised June 15, 2018; revised September 17, 2018; accepted January 30, 2019; published July 31, 2019

\begin{abstract}
This paper proposes a new measurement method for the effective measurement of the $99 \%$ occupied bandwidth (OBW) at monitoring stations. Although the OBW measurement of radio signal is recommended by the International Telecommunication Union Radio (ITU-R) with several methods, there still does not exist a clear measurement recommendation or standard for terrestrial DTV signal on-air environment. Modified adjacent channel power ratio (MACPR), which can be applied to 8-VSB (Vestigial Side Band) DTV (Digital Television) signal, is herein defined to verify the results of measurements obtained using the proposed measurement method. MACPR is a proper measuring parameter for determining the measuring area of a monitoring station. From measurement results obtained in real field environment, it has been found that the OBW of 8-VSB DTV signal can be effectively measured in areas where the MACPR value is over $35 \mathrm{~dB}$ and when the measurements are repeated more than 600 times in the same reception site. It also has been verified that measured results are within an error range of $+/-0.1 \%$ compared to results directly obtained at a transmission station. It is expected that the proposed method is able to be employed in order to determine the proper location of monitoring station and provide a reliable OBW measurement procedure for 8-VSB DTV signal on-air environment.
\end{abstract}

Keywords: Occupied Bandwidth Measurement, 8-VSB DTV Signal, Radio Monitoring, ACPR 


\section{Introduction}

Effective monitoring and management of radio signal by service providers is important for screening against illegal use of radio, identifying possible interferences between several services, and maintaining the quality of radio. Moreover, the management and monitoring of radio signal should be continuously and quickly performed, and monitoring technologies that have the confidence of service providers are required.

An occupied bandwidth (OBW) measurement, which is a type of radio monitoring method of terrestrial broadcasting, can be achieved by connecting measurement equipment directly to the radio frequency (RF) monitoring port of each broadcasting system. However, this method is problematic in that it is difficult to identify whether or not there is a violation of the OBW due to the fact that this method must give prior notice of the measurement to the broadcasting system. Thus, a radio monitoring method using OBW measurement should be performed in a real field, and the measured OBW must present a small error as well as a reliable level compared to the results obtained by directly connecting measurement equipment to the RF monitoring port of a transmission station. Although the OBW measurement of radio signal is recommended by the International Telecommunication Union Radio (ITU-R) with several methods, there still does not exist a clear measurement recommendation or standard for terrestrial DTV signal in a real field. Thus, we perform an OBW measurement of the Advanced Television System Committee (ATSC) terrestrial 8-VSB DTV broadcasting signal in a certain part of Seoul, Korea and propose a new measurement procedure and standards to effectively measure the OBW of terrestrial DTV signal on air .

This paper first performs a simulated measurement of generated 8-VSB DTV signal, in order to examine the characteristics of 8-VSB DTV signal and the reliability of the OBW measurement. The measurement results were used to make a standard for field measurement of terrestrial DTV signal. In addition, the measurement standard proposed in this paper was verified using statistical analysis of measurements made at monitoring stations and the direct measurement of the OBW using the RF monitoring port of a DTV transmission station.

In order to effectively measure 8-VSB DTV signal, this paper applies the 99\% OBW measurement method recommended by ITU-R and defines a modified adjacent channel power ratio (MACPR), which is a modified form of the existing adjacent channel power ratio (ACPR), for the configuration of a proper area for the measurement of the OBW in the field. While the 99\% OBW measurement method recommended by ITU-R does not refer to ACPR or MACPR for the measurement of the OBW, this paper proposes that MACPR, which is a modified form of ACPR that is used to compare the power of a transmitted channel with that of an adjacent channel to verify the linearity of a power amplifier in mobile communications, be used as a parameter to measure the $99 \%$ OBW. MACPR can be used as a parameter to determine a proper measuring area for performing highly reliable OBW measurements.

We also verify that the results of the OBW measurement made in the field using the measurement method proposed in this paper are not significantly different from the results of the direct measurement of the OBW using a RF monitoring port at a DTV transmission station. Thus, it is expected that the proposed method is able to be employed to effectively perform a reliable OBW measurement in real field.

A brief outline of the paper follows: In Section 2, the Z\% OBW is examined briefly. Section 3 presents the method for measuring the OBW of 8-VSB DTV signal at monitoring stations. Measurement results are then given in Section 4 for illustrating that reliable OBW 
measurements were achieved by the proposed measurement method with an error of less than $0.1 \%$ of the actual OBW of the transmitted signal.

\section{Z\% Occupied Bandwidth}

A bandwidth measurement method can be varied according to the type of bandwidth, such as absolute bandwidth, $3 \mathrm{~dB}$ bandwidth, equivalent bandwidth, null-to-null bandwidth, root mean square (RMS) bandwidth, Z\% occupied bandwidth, and $\mathrm{x} d \mathrm{~dB}$ bandwidth. ITU-R recommends Z\% occupied bandwidth for bandwidth measurement. The Z\% OBW presents an easy calculation and has the ability to specify a spectrum for various radio forms [1][2]. However, it does not have the ability to measure an exact OBW when interference signals that has strong magnetic field near the desired signal exist. Thus, the 8-VSB DTV signal employed in this paper was chosen as a measuring signal because there were no assigned channels around the 8-VSB DTV channel.

The Z\% OBW measurement method can be used to measure the width of a frequency band such that, above and below the upper and lower frequency limits, the average transmission power is each equal to $\beta / 2 \%$ of the total average power of a given signal for the span of a spectrum analyzer. If ITU-R does not present a specific reference for a special radio form, the value of $\beta / 2 \%$ is equal to 0.5 . Fig. 1 shows the definition of the Z\% OBW recommended by ITU-R. The $99 \%$ OBW is herein employed as a measurement standard in which the value of $\beta / 2 \%$ is 0.5 [3].

Of particular note in the calculation of the Z\% OBW of an 8-VSB DTV signal is that a pilot signal exists in 8-VSB DTV signal. Because the Z\% OBW is defined as the bandwidth excluding the values to the left and right of $\beta / 2 \%$ of the total transmission average power in a spectrum, which presents a symmetric shape in its left and right sections, the exact OBW can be measured by using the total average transmission power which is calculated by excluding the pilot signal in the 8-VSB DTV signal.

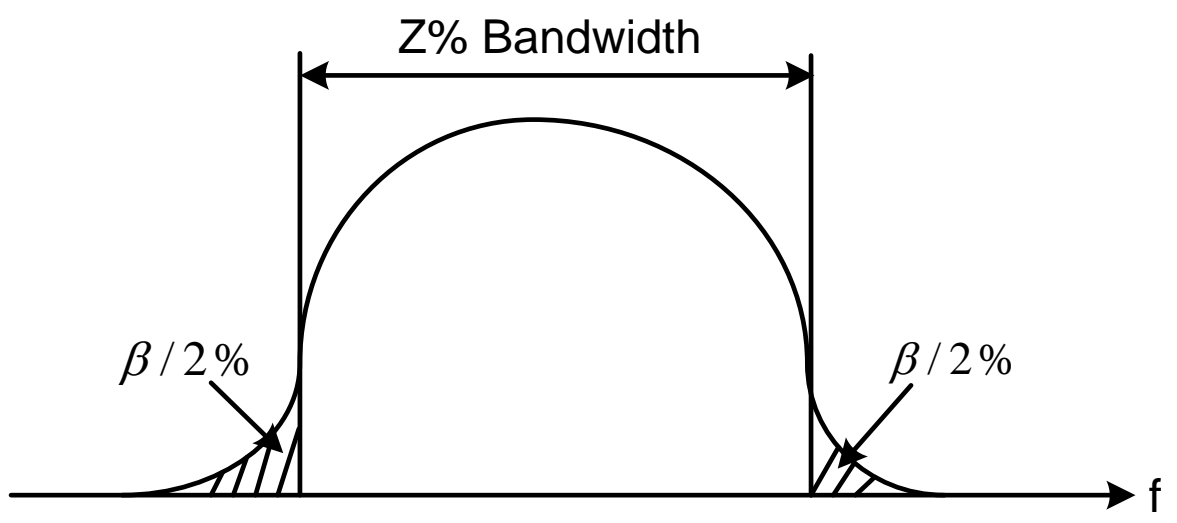

Fig. 1. Definition of the Z\% occupied bandwidth 


\section{Proposed Measurement Method}

\subsection{Measurement System and Procedures}

Fig. 2 shows the configuration of a measurement system, which is able to measure 8-VSB DTV signal at a monitoring station. The major measuring equipment used in this work consisted of a spectrum analyzer, a log periodic (LP) antenna, a global positioning system (GPS) satellite receiver, a control system, which should be able to control a receiver antenna, a storing and analyzing system for the received DTV signal data, and other auxiliary equipment.

The major characteristics of a LP antenna are given in Table 1. The connection cable between the LP antenna (a type of receiver antenna) and the spectrum analyzer was a RG-8 coaxial cable with a length of $10 \mathrm{~m}$ and loss of $0.7 \mathrm{~dB}$. A GPS satellite receiver was used to measure the latitude and longitude of the measurement area. The major measurement parameters employed in this paper are given in Table 2. In Table 2, the number of pixels is the unique number of pixels used in the spectrum analyzer to measure the OBW. Since the resolution bandwidth (RBW) is to be taken as approximately less than 3\% of the span, the transmission bandwidth of 8-VSB DTV signal can be exactly measured when the RBW is configured to be less than $30 \mathrm{kHz}$ [4]. In the reference [4], $30 \mathrm{kHz}$ measurement bandwidth, i.e. RBW of spectrum analyzer, has a small amount of error near the band edge [4]._Thus, the value of the RBW was taken as $30 \mathrm{kHz}$. Since the video bandwidth (VBW) can usually be defined as more than three times the RBW, the value of the VBW was taken as $300 \mathrm{kHz}$, which is 10 times the RBW to maximally reduce a peak error of the spectrum analyzer [5][6]. The span was taken as $12 \mathrm{MHz}$, double the DTV channel bandwidth of $6 \mathrm{MHz}$, in order to apply MACPR, which is one of the major factors in measuring the OBW [7]. The parameters shown in Table 2 are the optimal parameters to effectively measure the OBW of 8-VSB DTV signal.
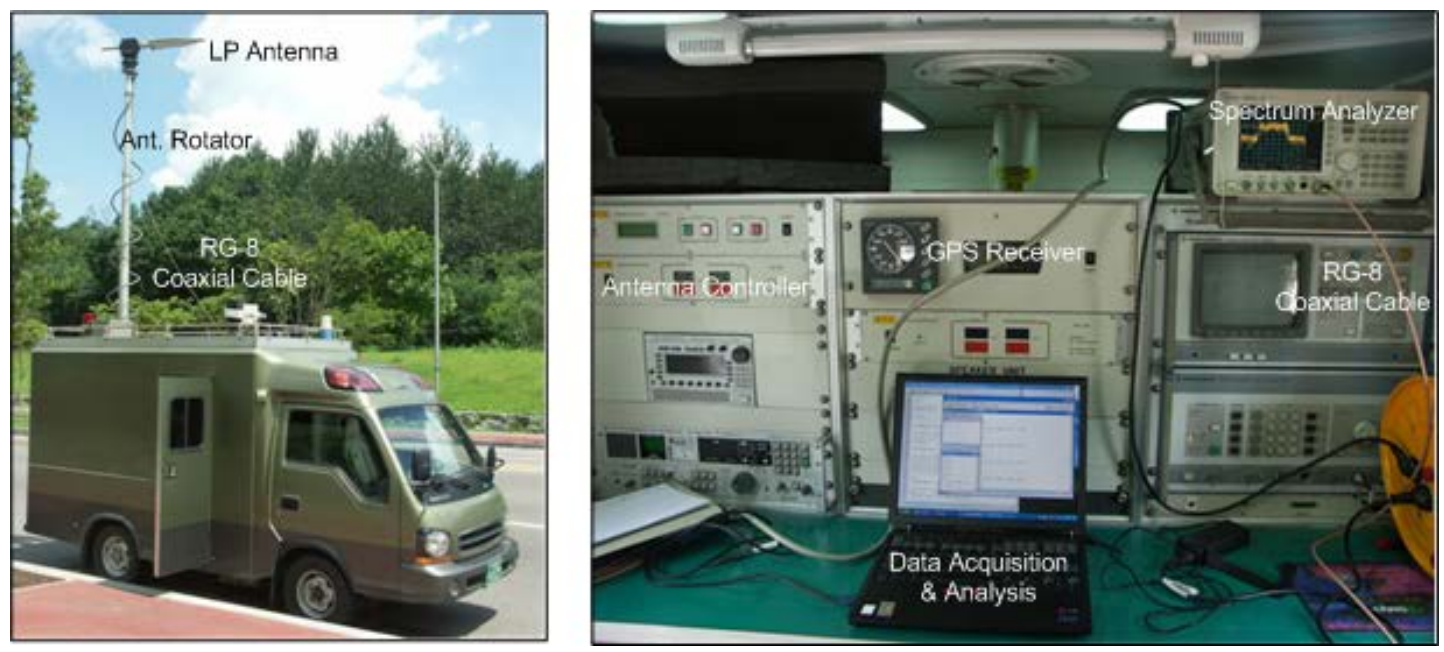

Fig. 2. Measurement system used at a mobile monitoring station 
Table 1. MAJOR CHARACTERISTICS OF A LP ANTENNA

\begin{tabular}{cc}
\hline \hline Parameter & Value \\
\hline Antenna Type & Log Periodic Dipole \\
Frequency & $400 \mathrm{MHz} \sim 3 \mathrm{GHz}$ \\
Gain & $5 \sim 8 \mathrm{dBi}$ \\
Front-back Ratio & $15 \mathrm{~dB}$ \\
\hline
\end{tabular}

Table 2. MAJOR PARAMETERS OF A SPECTRUM ANALYZER

\begin{tabular}{cc}
\hline \hline Parameter & Value \\
\hline Number of Pixels & 601 \\
RBW & $30 \mathrm{kHz}$ \\
VBW & $300 \mathrm{kHz}$ \\
Sweep Time & $50 \mathrm{~ms}$ \\
Span & $12 \mathrm{MHz}$ \\
Detection Mode & Sample Mode \\
Average Mode & OFF \\
\hline
\end{tabular}

\subsection{Definition of MACPR}

Because the Z\% OBW is defined as the bandwidth equivalent to $99 \%$ of the power of the measured channel, the OBW can be varied according to the power of the measured channel. Thus, the measurement should be made in a region where the channel power is guaranteed at a certain level in order to apply the Z\% OBW measurement method in the field. However, there are no specific parameters which is needed for the determination of the location of a monitoring station and the effective measurement of the Z\% OBW recommended by ITU-R. Therefore, for the application of Z\% OBW in a real field, we propose MACPR as a parameter to choose the proper location of a monitoring station where a certain reliable OBW is to be measured.

MACPR is a modified type of ACPR calculation, which is shown in Fig. 3. ACPR is used as a standard to present the linearity of a power amplifier in mobile communications [8]. Because ACPR represents the ratio of the transmitted channel power to the adjacent channel power, the ACPR means a change in the transmitted channel power if the adjacent channel power is changed slightly. Thus, it is possible to define a parameter that is useful for determining a region to measure the $\mathrm{Z} \% \mathrm{OBW}$ in the case of proper application of ACPR.

As shown in Fig. 4, we define MACPR, a modified type of the existing ACPR calculation, and propose a parameter to determine a certain OBW measuring area for 8-VSB DTV signal. The existing ACPR corresponds to the calculation using the power of the total channel bandwidth divided by the power of the $30 \mathrm{kHz}$ bandwidth of the adjacent channel [8]. However, the MACPR is defined as the ratio of the power within the frequency band of $\left[f_{c}-3 \mathrm{MHz}, f_{c}+3 \mathrm{MHz}\right]$ to the power within the lower half adjacent channel $\left[f_{c}-6 \mathrm{MHz}, f_{c}-3 \mathrm{MHz}\right]$ and upper half adjacent channel $\left[f_{c}+3 \mathrm{MHz}, f_{c}+6 \mathrm{MHz}\right]$. In the previous notation, fc denotes the center frequency of the transmitted signal and $[a, b]$ denotes the range between $a$ and $b$. 


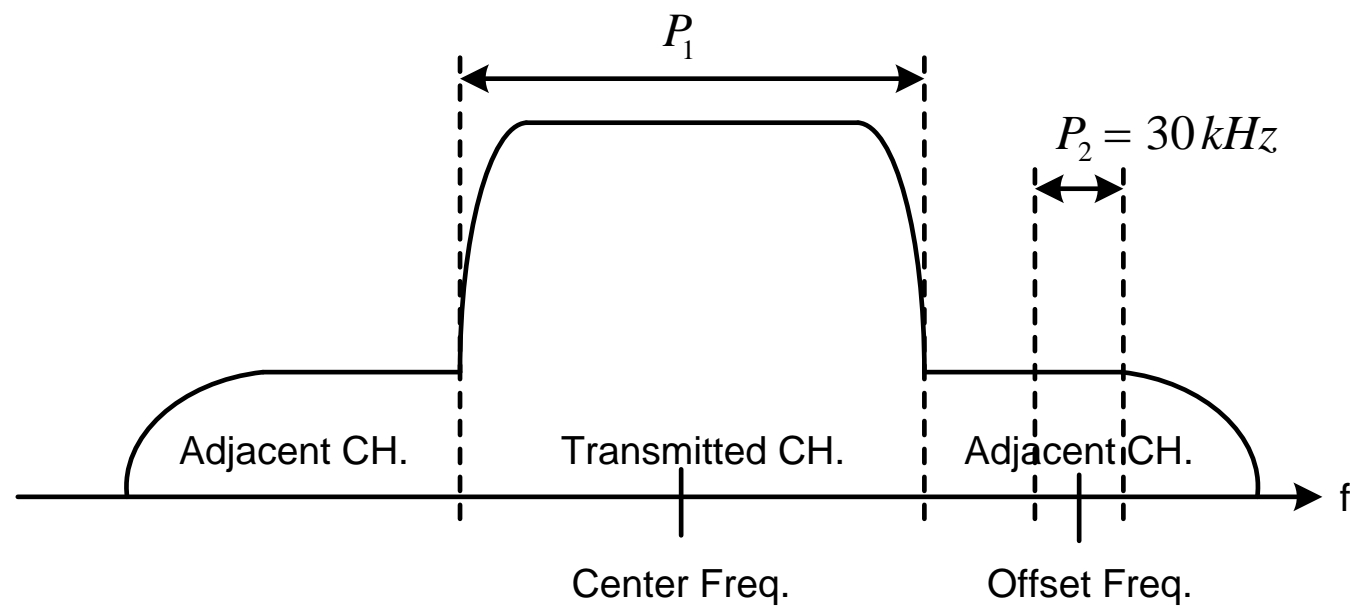

Fig. 3. Definition of ACPR

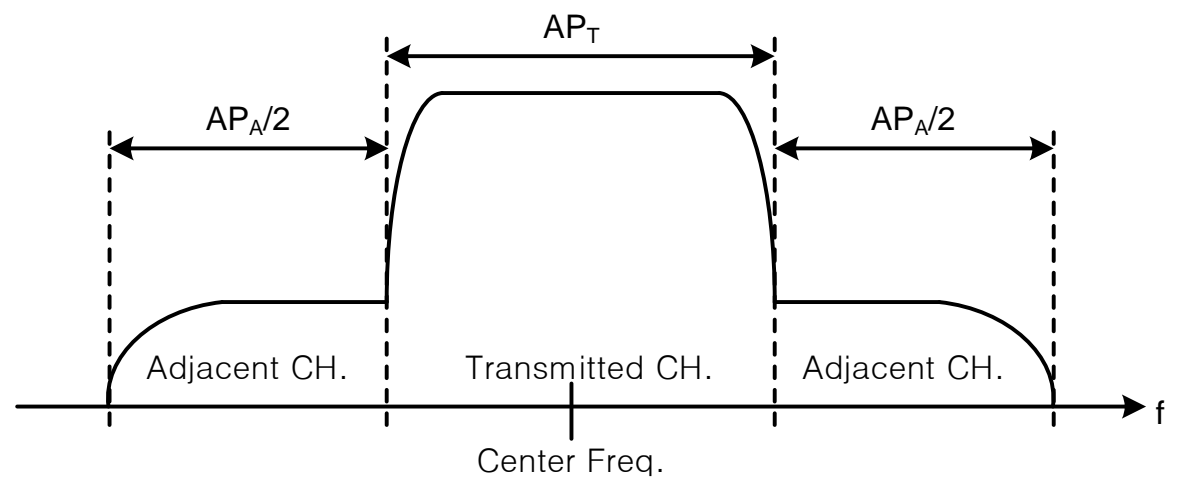

Fig. 4. Definition of MACPR

$$
A C P R=10 \log \left(\frac{P_{1}}{P_{2}}\right)
$$

where $P_{1}$ is the power within a transmitted channel and $P_{2}$ is the power within a bandwidth of $30 \mathrm{kHz}$ in an adjacent channel.

$$
M A C P R=10 \log \left(\frac{A P_{T}}{A P_{A}}\right)
$$

where $A P_{T}$ is total power measured in a $6 \mathrm{MHz}$ in-channel bandwidth and $A P_{A}$ is total power measured in two $3 \mathrm{MHz}$ adjacent channel bandwidth.

Since MACPR can be used to identify the power ratio of the transmission channel to the adjacent channel, it is possible to effectively represent changes in the power of the transmission channel due to environmental factors in the measurement of 8-VSB DTV signal in the field, such as multipath loss and a decrease in the magnetic field strength due to an increase in the distance from a transmission station. On the other hand, if the adjacent channel 
no longer exists, $A P_{A}$ is the measurement instrument's own noise power in the adjacent channel. So, MACPR can be used to identify the power ratio of the transmission channel to the measurement instrument's own noise.

\subsection{Relationship between MACPR and noise floor}

In this section, the impacts of noise floor on the proposed MACPR parameter are examined under the condition that the adjacent channel is not in use on the air. The reason is that our proposed measurement method is applicable to the measurement of the OBW of the transmitted channel whose the adjacent channels are vacant. The configuration of the measurement system in a monitoring station is illustrated as shown in Fig. 5[9].

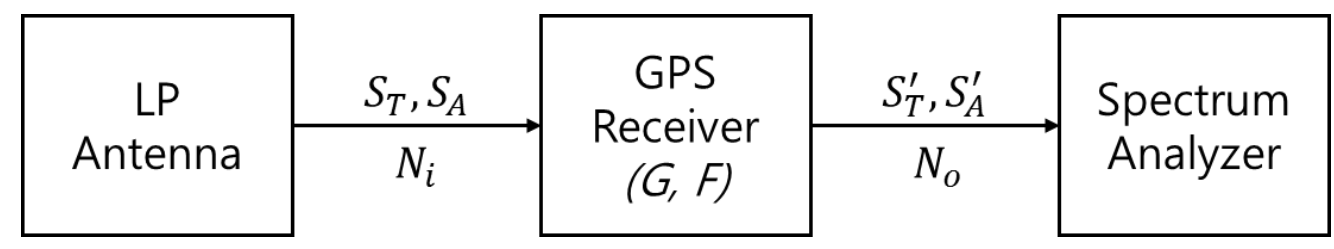

Fig. 5. The configuration of measurement system

From Fig. 5, it is observed that No, $\mathrm{AP}_{\mathrm{T}}$ and $\mathrm{AP}_{\mathrm{A}}$ can be expressed as

$$
\begin{aligned}
& \mathrm{N}_{\mathrm{o}}=G \cdot N_{i} \cdot F \\
& \mathrm{AP}_{\mathrm{T}}=S_{T}^{s}+N_{o}=G S_{T}+N_{o} \\
& \mathrm{AP}_{\mathrm{A}}=S_{A}^{j}+N_{o}=G \mathrm{~S}_{\mathrm{A}}+N_{o}
\end{aligned}
$$

in which $\mathrm{N}_{\mathrm{i}}$ and $\mathrm{N}_{\mathrm{o}}$ are the noise powers at the amplifier input and output ports, respectively, $\mathrm{G}$ is the amplifier gain, $\mathrm{F}$ is a noise figure, $\mathrm{S}_{T}$ and $\mathrm{S}_{T}^{\prime}$ are the powers of the transmitted channel at the amplifier input and output ports, respectively, and $S_{A}$ and $S_{A}^{\prime}$ are the powers of the adjacent channel at the amplifier input and output ports, respectively.

In the expression (3), the same noise power No is added to each channel since the bandwidth of the adjacent channel is equal to that of the transmitted channel. Furthermore, $\mathrm{S}_{\mathrm{A}}^{\prime}$ is taken as zero since the adjacent channel is vacant.

Upon substituting Eq. (3) into Eq. (2) and taking $\mathrm{S}_{\mathrm{A}}^{\prime}$ as zero, MACPR is given by

$$
\text { MACPR }=10 \log \frac{A P_{T}}{A P_{A}}=10 \log \frac{G-S_{T}+N_{0}}{N_{0}}=10 \log \frac{S_{T}}{N_{i} \cdot F}+1 \approx 10 \log \frac{S_{T}}{N_{i}}-\log F
$$

From the expression (4), it can be seen that MACPR is nearly proportional to SNR $\frac{S_{T}}{N_{i}}$ if SNR is relatively large enough to neglect the noise floor $\mathrm{N}_{\mathrm{i}}$. This implies that the environmental factors such as fading margin and the noise characteristics of the receiver do not have significant impacts on the accuracy of the OBW of the transmitted channel which is made a measurement at the location where MACPR is relatively large.

One simulated test has been carried out in the lab in order to demonstrate the proposed parameter MACPR is not sensitive to the strength of noise floor in case of relatively large SNR.

Fig. 6 shows the changes in the power of an 8-VSB DTV transmission channel and an adjacent channel according to changes in MACPR. While the power of the 8-VSB DTV 
transmission channel varied linearly with the MACPR value, the power of the adjacent channel was not significantly changed. Therefore, it is possible to apply MACPR to the identification of a proper reception site for measurement of the OBW because the MACPR value is proportional to the magnetic field strength of the 8-VSB DTV channel and it is also inversely proportional to the distance between the transmitter and the monitoring station. Also, Fig. 6 represents the difference between the instrument's own noise power and the power of the 8-VSB DTV signal. As the adjacent channel no longer exists, the adjacent channel in the Fig. 6 can stand for instrument's own noise power.

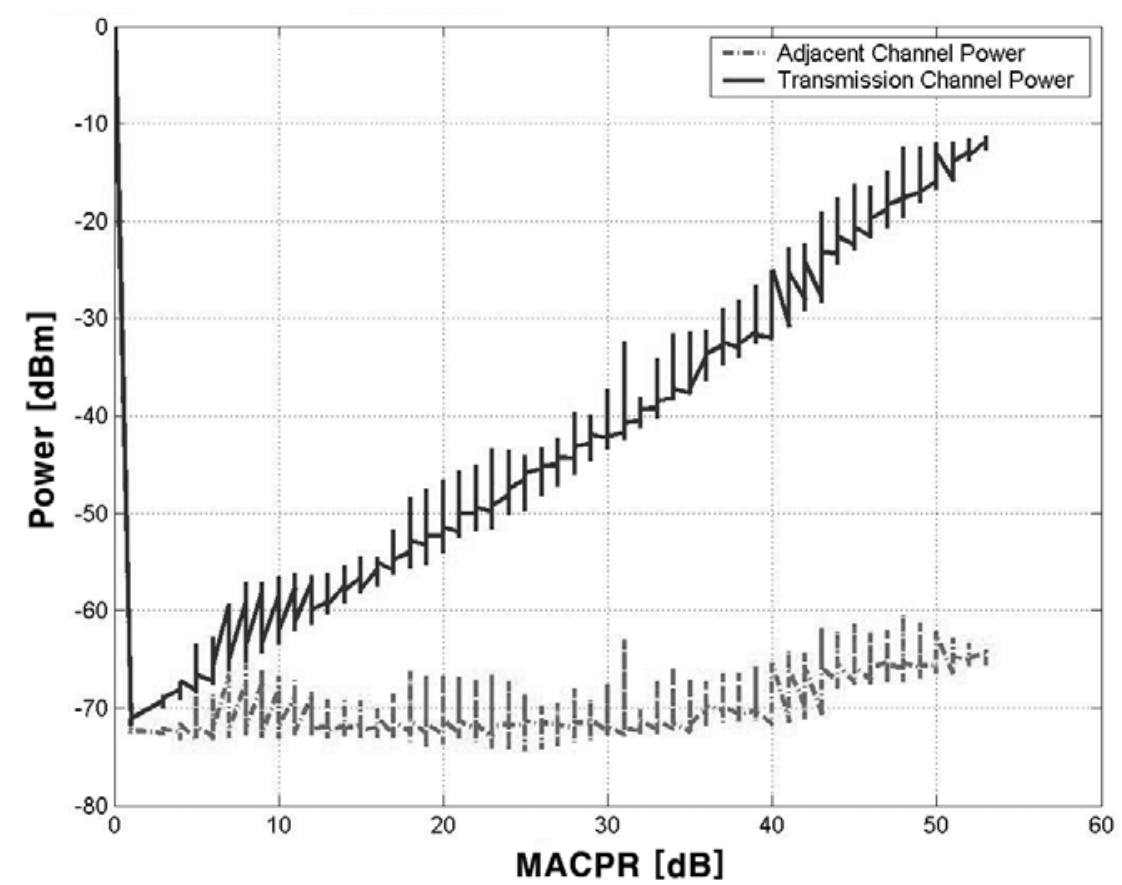

Fig. 6. Power comparison of an 8-VSB DTV transmitted channel to an adjacent channel as a function of the MACPR value

\subsection{Optimum number of measurement trials}

Multiple measurements of the OBW using a spectrum analyzer in the field may not present the same results due to rapid change in radio environmental factors, even though the measurements are made at the same site. In addition, the measured spectrum can be slightly changed due to nonlinear characteristics of the spectrum analyzer. It is impossible to estimate these changes, and a small number of measurement trials are not sufficient for real, reliable measurements. Hence, the measurement of the OBW can be made using a statistical method with many measurement trials [10].

The distribution of the 99\% OBW obtained from measured spectrum data, which can be produced by repeating the measurement more than 1,000 times using a spectrum analyzer in the field, follows the statistical characteristics of a Gaussian distribution and is shown in Fig. 7. Thus, the configuration of the OBW in a real field can be achieved using statistical methods 
that follow Gaussian distributions. Fig. 8 shows a standard normal distribution, which follows a Gaussian distribution, and the number of optimum measurement trials can be defined by Eq. (5) [11].

$$
N=\left(\frac{Z_{\alpha / 2} \times \sigma}{e}\right)
$$

where, $N$ is the number of proper samples, $Z_{\alpha / 2}$ is a standard normal distribution constant based on the reliability level, $\sigma$ is a standard deviation, and e is an error range.

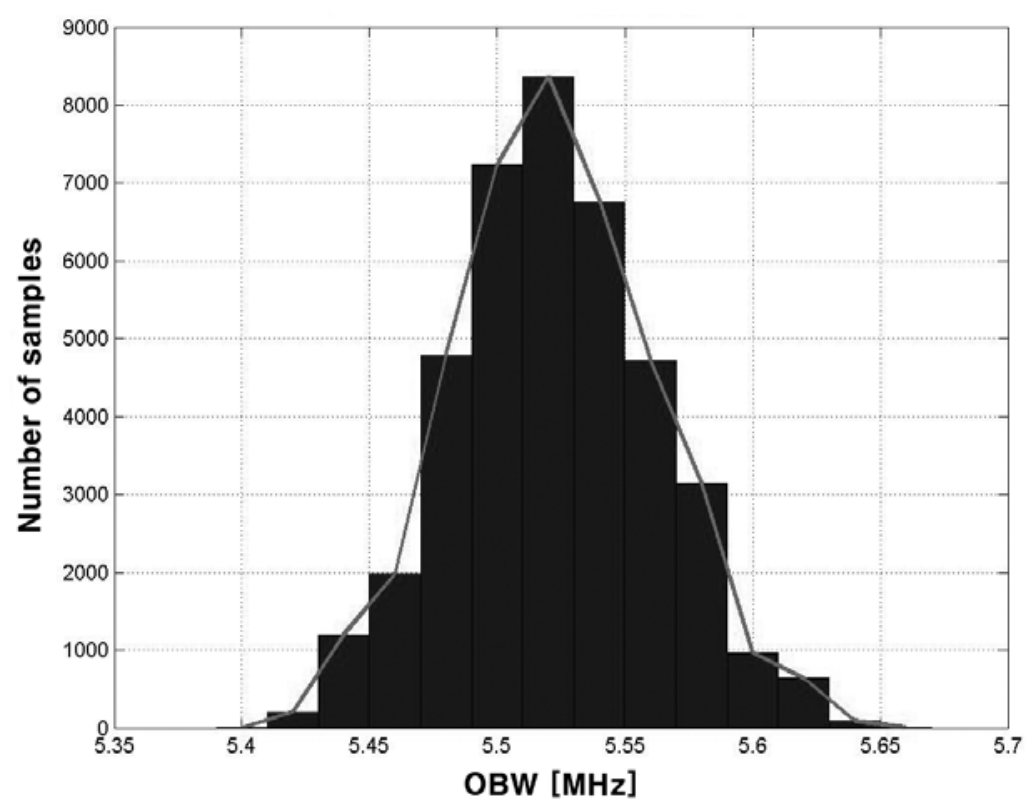

Fig. 7. Occupied bandwidth distribution of DTV signal measured in a real field

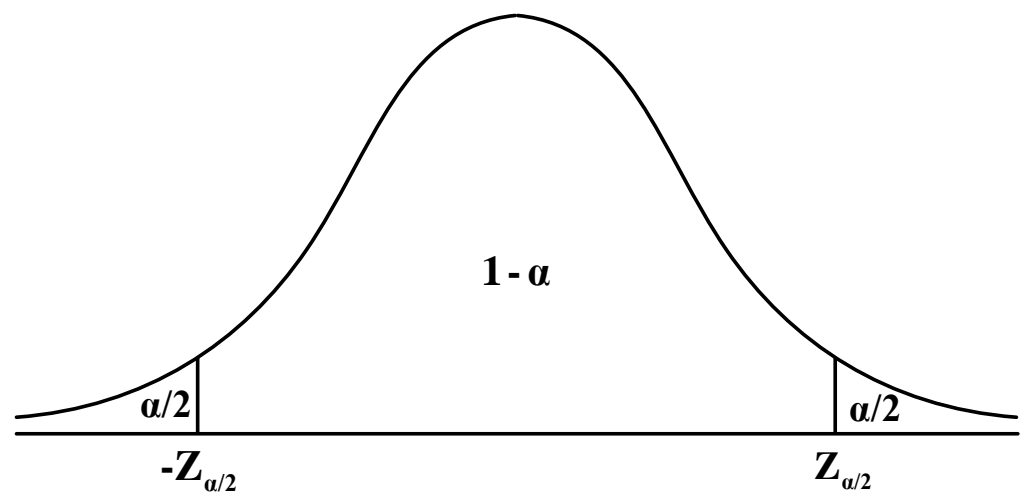

Fig. 8. Standard normal distribution

Thus, the number of samples used to measure the OBW of 8-VSB DTV signal (i.e. the number of measurements) can be defined by the reliability level, error range, and standard deviation. In the next section, we will define the number of optimum measurement trials to provide a reliability level of $99 \%$ and an error range of $+/-0.1 \%$. 


\section{Measurement Results}

Virtual 8-VSB DTV signal were generated and used for the development of an OBW measuring standard; the measuring standard was derived through simulation and subsequent statistical analysis. The validity of this measuring standard was also verified by applying it to a real field. Actual field measurements of 8-VSB DTV signal were classified as measurement of the transmission signal at the monitoring port of a transmission station and field measurement at monitoring stations.

\subsection{Measurement results using simulated 8-VSB DTV signal}

To consider the effect of MACPR on the OBW of 8-VSB DTV signal and to investigate how MACPR varies with magnetic field strength, simulated DTV signal were generated and a simulation was performed. The modulation type was 8-VSB and the bandwidth was taken as $5.5223 \mathrm{MHz}$.

Changes in relative error and average OBW were obtained as a function of the change in the MACPR value in order to determine an exact value of the MACPR that could provide a relative error of $0.1 \%$ of the bandwidth of $5.5223 \mathrm{MHz}$ of the simulated 8-VSB DTV signal in the simulated tests. Fig. 9 shows the changes in the average OBW according to changes in the MACPR value, and Fig. 10 shows the changes in relative error according to changes in the MACPR value. As a result, it can be seen that the relative error is within $+/-0.1 \%$ for a bandwidth of $5.5223 \mathrm{MHz}$ when the MACPR value is taken as more than $35 \mathrm{~dB}$. As examined in section 3.3, MACPR of $35 \mathrm{~dB}$ is large enough to neglect the effect of noise floor. Therefore, we shall take MACPR value as $35 \mathrm{~dB}$ for effectively determining the location of monitoring station on-air environment.

In addition, because the measured OBW has statistical characteristics of a standard normal distribution following a Gaussian distribution, it is possible to obtain the minimum number of measurement samples, which are to be configured with a reliability level of $99 \%$ and an error of $+/-0.1 \%$. The number of proper samples obtained from Eq. (5) was 341 .

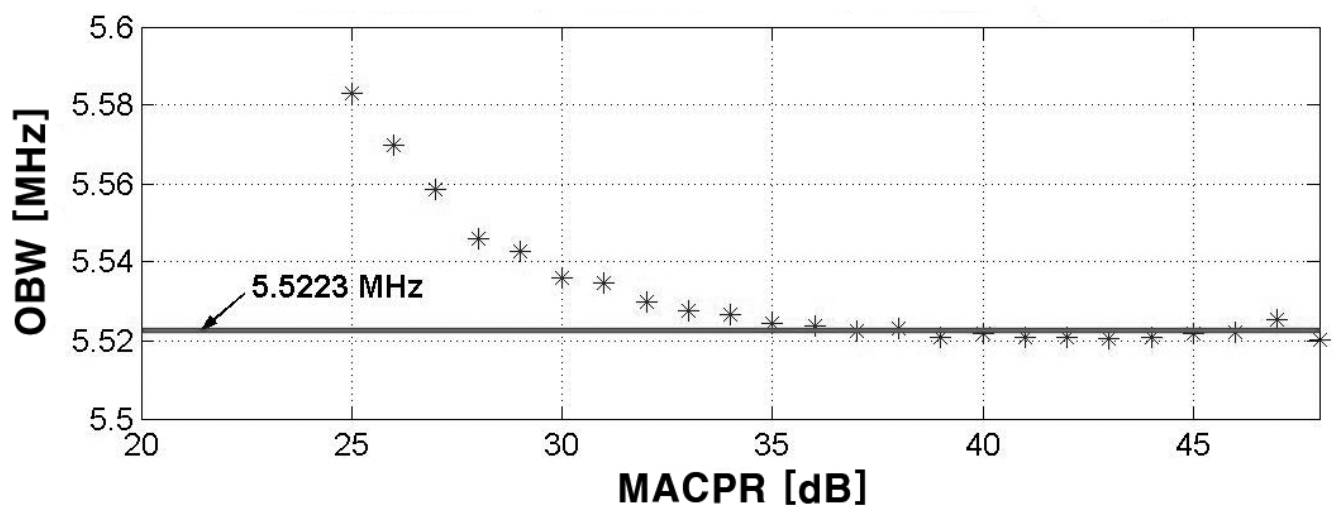

Fig. 9. Changes in average occupied bandwidth as a function of the MACPR value 
The 99\% OBW measurement method recommended by ITU-R was applied to a region with MACPR value of more than $35 \mathrm{~dB}$. In this case, it was possible to measure a highly reliable OBW when the minimum number of measurements trials was 341. In the real field measurement, the number of measurement trials was set at more than 600 to increase the reliability of the results.

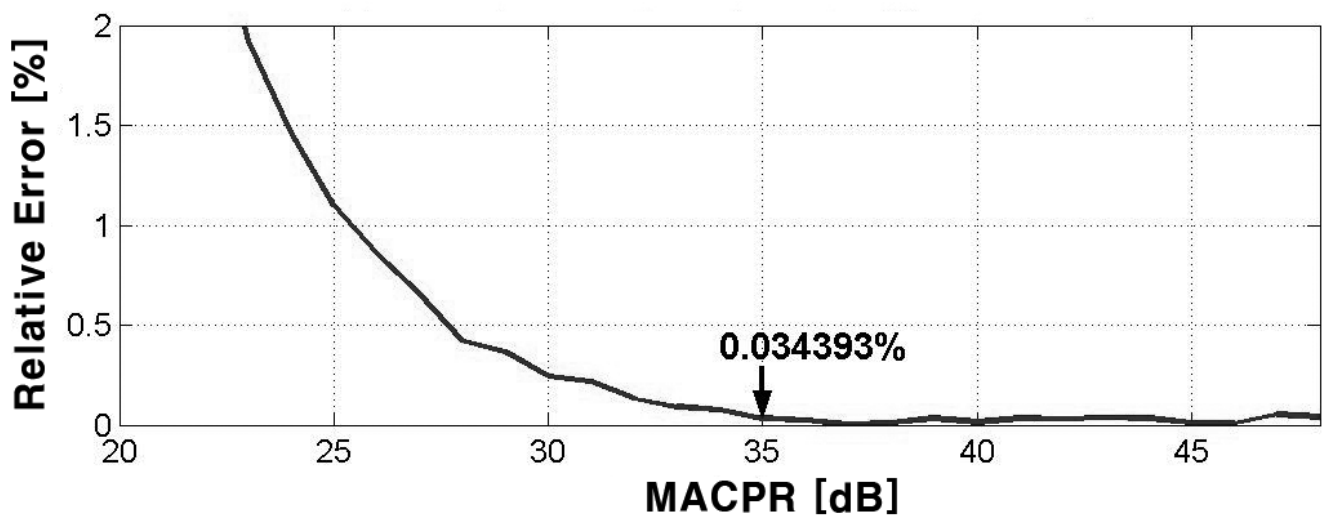

Fig. 10. Changes in relative error as a function of the MACPR value

\subsection{Measurement results at the DTV transmission station}

Transmitted signal were measured at the monitoring port of an 8-VSB DTV transmission

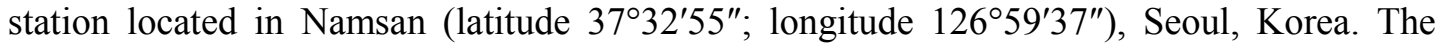
center frequency of the transmitted DTV signal was $797 \mathrm{MHz}$. The signal was measured 1,000 times by connecting a spectrum analyzer to the RF monitoring port of the transmission station.

Radio waves are reflected, absorbed, scattered, refracted, and diffracted by the atmospheric conditions that they encounter, such as clouds and precipitation. Critically, different atmospheric conditions impact radio waves differently. The signals of commercial terrestrial users of spectrum, like cell towers or broadcasting relay towers, are often much stronger or weaker than the signals being transmitted due to the weather, water, and climate communities. This can cause radio frequency interference (RFI) with weather, water, and a variety of climate, which can degrade signal detection. Thus, the measurement was herein made in clear weather to minimize the deleterious problems due to bad case of weather condition and obtain the reliable results being employed for the OBW measurement of the DTV signals in real field.

Table 3 shows the results for the MACPR and the OBW measured at the transmission station. From the results, the average OBW was $5.4785 \mathrm{MHz}$, the maximum and minimum OBW were $5.58 \mathrm{MHz}$ and $5.36 \mathrm{MHz}$, respectively, and the standard deviation was $39.489 \mathrm{kHz}$. In addition, the average MACPR was $51.511 \mathrm{~dB}$. 
Table 3. OCCUPIED BANDWIDTH AND MACPR MEASURED AT THE DTV TRANSMISSION STATION

\begin{tabular}{cc}
\hline \hline Parameter & Value \\
\hline Measurement Trials & 1,000 \\
Occupied Bandwidth & $5.4785 \mathrm{MHz}$ \\
Standard Deviation & $39.489 \mathrm{kHz}$ \\
Maximum OBW & $5.58 \mathrm{MHz}$ \\
Minimum OBW & $5.36 \mathrm{MHz}$ \\
Mean MACPR & 51.511 \\
\hline
\end{tabular}

\subsection{Measurement results at monitoring station}

Measurements in the field were made $600 \sim 1,000$ times for every measuring region after selecting 43 measuring areas that were guaranteed to be Line-of-Sight (LOS) environments in order to minimize decrease in magnetic field strength due to multi-path loss or environmental factors. Fig. 11 shows the 43 measuring areas in Seoul, Korea.

Fig. 12 shows the measurement results for the OBW obtained in a real field with a choice of the MACPR value. As shown in Fig. 12, the OBW in a real field converges to a certain constant level of the OBW in which the MACPR has a higher value than a certain specific level. Therefore, it can be seen that the measured result approaches the value of the OBW directly measured at the RF monitoring port of the 8-VSB DTV transmission station when the $\mathrm{OBW}$ is measured at a region where the error range was within $+/-0.1 \%$ and the MACPR was more than $35 \mathrm{~dB}$. Fig. 13 shows the OBW distribution and statistical characteristics of the sample data obtained in the field. It can be seen in Table 4 that the average OBW of the measured data (MACPR value of more than $35 \mathrm{~dB}$ ) was $5.4816 \mathrm{MHz}$, the maximum and minimum OBW were $5.68 \mathrm{MHz}$ and $5.32 \mathrm{MHz}$, respectively, and the standard deviation was $49.436 \mathrm{kHz}$. Thus, the average OBW for a MACPR value over $35 \mathrm{~dB}$ did not exceed an error range of $+/-0.1 \%(0.0566 \%)$ of the average OBW of $5.4785 \mathrm{MHz}$ measured at the DTV transmission station. This implies that the assumptions made in the simulated tests, where the OBW can be measured within an error range of $+/-0.1 \%$ and a MACPR value over $35 \mathrm{~dB}$, can also be applied to a real field.

The average OBW obtained from random sampling of over 600 samples among the measured samples with MACPR $\geq 35 \mathrm{~dB}$ was $5.4809 \mathrm{MHz}$, the maximum and minimum OBW were $5.62 \mathrm{MHz}$ and $5.34 \mathrm{MHz}$, respectively, and the standard deviation was $50.921 \mathrm{kHz}$. As a result, the average OBW did not exceed the desired error range of $+/-0.1 \%(0.0438 \%)$ of the average OBW of $5.4785 \mathrm{MHz}$ measured at the DTV transmission station. Therefore, the OBW obtained from the measured samples, which were repeatedly obtained more than 600 times with MACPR values over $35 \mathrm{~dB}$, are within an error range of $+/-0.1 \%$ of the OBW directly obtained at the DTV transmission station. 


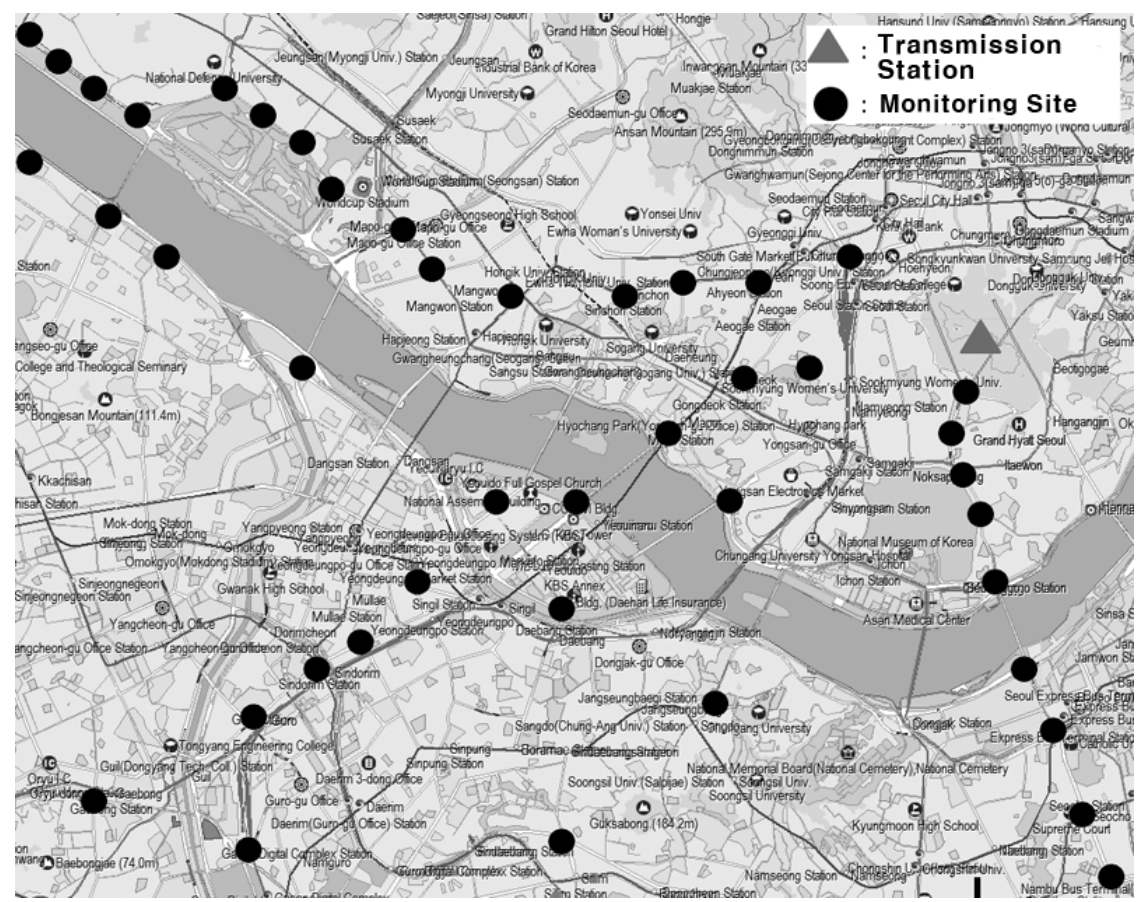

Fig. 11. The 43 measurement sites for OBW measurement; The triangle point is the DTV transmission station and the black points are the monitoring sites.

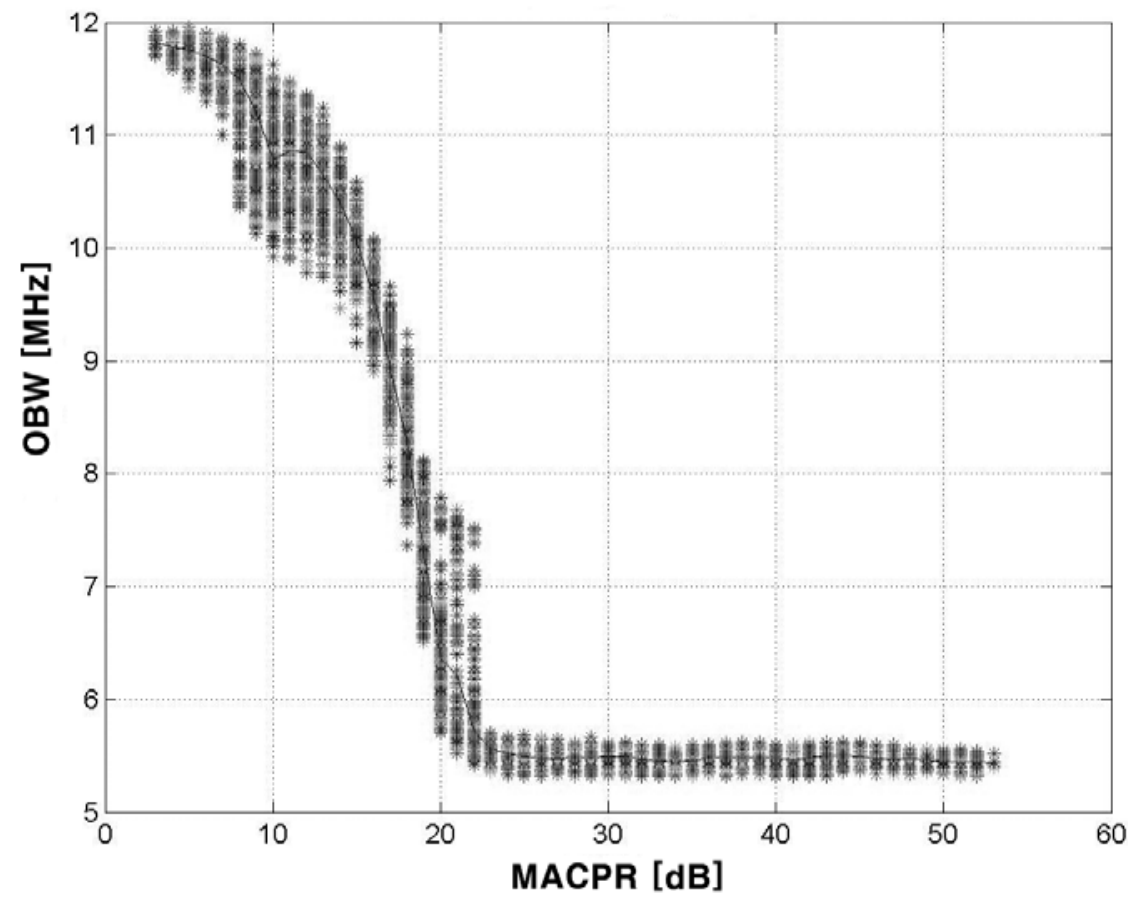

Fig. 12. Occupied bandwidth vs. MACPR at monitoring stations 


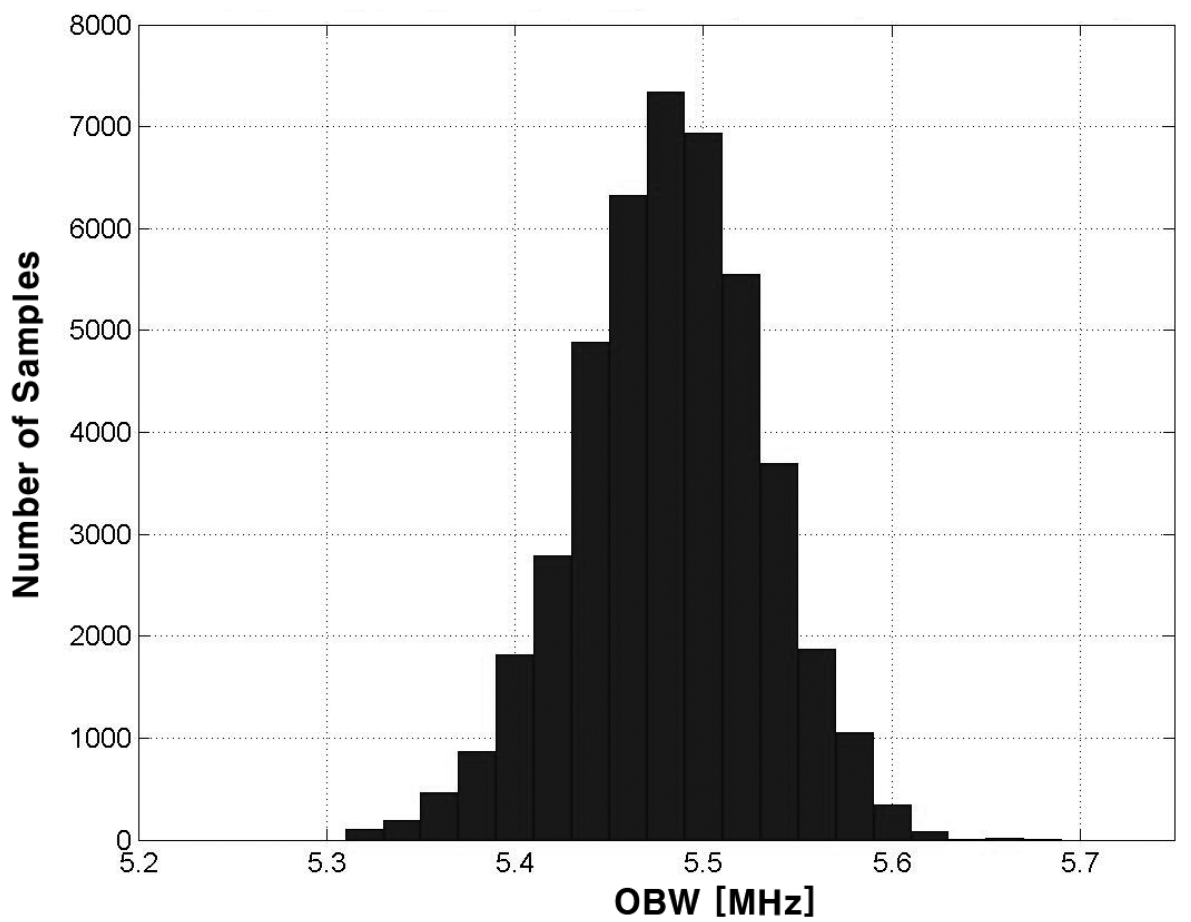

Fig. 13. Distribution of occupied bandwidth with $M A C P R \geq 35 \mathrm{~dB}$ obtained from measurements at monitoring stations

Table 4. Comparison of Statistical OBW Measurement results between

\begin{tabular}{|c|c|c|c|}
\hline \multirow{2}{*}{$\begin{array}{l}\text { Parameter } \\
\text { Measurement } \\
\text { Trials }\end{array}$} & \multirow{2}{*}{$\begin{array}{c}\text { Transmission } \\
\text { Station }\end{array}$} & \multicolumn{2}{|c|}{$\begin{array}{l}\text { Monitoring Station with } \\
\text { MACPR } \geq 35 \mathrm{~dB}\end{array}$} \\
\hline & & 44,335 & 600 \\
\hline $\begin{array}{l}\text { Occupied } \\
\text { Bandwidth[MHz] }\end{array}$ & 5.4785 & 5.4816 & 5.4809 \\
\hline $\begin{array}{l}\text { Standard } \\
\text { Deviation }[\mathrm{kHz}]\end{array}$ & 39.489 & 49.436 & 50.921 \\
\hline $\begin{array}{l}\text { Maximum } \\
\text { OBW[MHz] }\end{array}$ & 5.58 & 5.68 & 5.62 \\
\hline $\begin{array}{l}\text { Minimum } \\
\text { OBW[MHz] }\end{array}$ & 5.36 & 5.32 & 5.34 \\
\hline Relative Error[\%] & - & 0.0566 & 0.0438 \\
\hline
\end{tabular}

The measurement parameters and procedure are summarized as follows:

- Measurement conditions and antenna parameters:

- Line of sight transmission between the transmitter and receiver antenna must be ensured.

- A directional antenna is recommended to reduce the effect of multipath fading and interferences with the OBW measurement. 
- Measurement parameters for the spectrum analyzer:

- Center frequency: Carrier frequency of the transmitter

- Resolution bandwidth: $30 \mathrm{kHz}$

- Video bandwidth: $300 \mathrm{kHz}$

- Scan bandwidth (SPAN): $12 \mathrm{MHz}$

- Sweep time: $50 \mathrm{~ms}$

- Detection mode: Sample mode

- Measurement procedure:

- Step 1: Calculate MACPR in a scan bandwidth of $12 \mathrm{MHz}$.

- Step 2: Calculate 99\% OBW for that MACPR value, and record both OBW and MACPR.

- Step 3: Repeat step 1 and 2 for more than 600 measurements of the OBW for MACPR values greater than $35 \mathrm{~dB}$.

- Step 4: Acquire an average OBW and compare that with the prescribed OBW.

\section{Conclusion}

In this paper, we have proposed an effective measurement method of the OBW for 8-VSB DTV signal at monitoring stations. While the OBW measurement methods recommended by the ITU-R are not applicable to terrestrial DTV signal on-air environment, the proposed metbod can be employed to measure the OBW of DTV signal on-air since it provides the reliable results on comparing with the direct measurement at the transmission station. When the proposed method was employed for 99\% OBW measurements at monitoring stations with MACPR values over $35 \mathrm{~dB}$, it was found that the results in the field were very close to those obtained at the transmission station and were within an error of $+/-0.1 \%$ relative to the average 99\% OBW obtained from the direct measurement at the RF monitoring port of the transmission station. Furthermore, it has been found that the environmental factors such as fading margin and the noise characteristics of the receiver do not have significant impacts on the accuracy of the OBW of the transmitted channel which is made a measurement at the location where MACPR is relatively large (e.g., over $35 \mathrm{~dB}$ ). The proposed measurement method is expected to provide reliable results for 99\% OBW measurements of 8-VSB DTV signal on the air.

In future work, we shall consider whether the proposed measurement method is effectively applicable to several types of the modulated signals in real field.

\section{References}

[1] Recommendation Spectrum Management SM.433: Methods for the Measurement of Radio Interference and the Determination of Tolerable Levels of Interference, ITU-R Recommendation, June 2003.

[2] Spectrum Monitoring Handbook, Edition 2002, ITU-R, pp.257-274, 2002.

[3] Recommendation Spectrum Management SM.443: Bandwidth Measurement at Monitoring Stations, ITU-R Recommendation, June 2003.

[4] Linley Gumm, "Measurement of 8-VSB DTV transmitter emissions," IEEE Trans. Broadcasting, vol. 45, no. 2, pp.234-242, June 1999. 
[5] Carl Eilers and Gary Sgrignoli, "Digital television transmission parameters analysis and discussion,” IEEE Trans. Broadcasting, vol. 45, no. 4, pp.365-385, Dec. 1999. Article (CrossRef Link)

[6] Rohde \& Schwarz Application Note: Swept Adjacent Channel Power Analysis on Digital TV Amplifiers, Rohde \& Schwarz, 2001.

[7] Tektronix Application Note: 8 VSB Measurements Using the RFA300A, Tektronix, 2001.

[8] Kevin G. Gard, Hector M. Gutierrez and Michael B. Steer, "Characterization of spectral regrowth in microwave amplifiers based on the nonlinear transformation of a complex Gaussian process," IEEE Trans. Microwave Theory and Techniques, vol. 47, no. 7, pp. 1059-1069, July 1999. Article (CrossRef Link)

[9] B. Sklar, "Digital Communications Fundamentals and Applications," Prentice Hall, New Jersey, $2^{\text {nd }}$ Edition, 2001.

[10] Jay L. Devore, "Probability \& Statistics for Engineering and the Sciences,” Wadsworth, 317-342, 1982.

[11] Wayne W. Daniel and James C. Terrell, "Business Statistics,” Houghton Mifflin, pp.232-252, 1989.

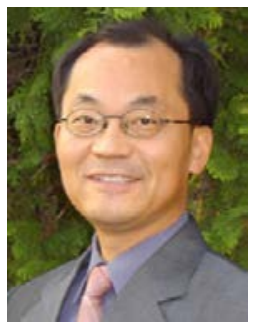

Dr. Young Soo Kim received the B.S. and M.S. degrees in electronic engineering from Yonsei University in 1981 and 1983, respectively, and the Ph.D. degree in electrical and computer engineering from Arizona State University, Tempe, in 1988.

In May 1985, he joined the Signal-System Technology Inc., U.S.A., where he did research on sonar signal processing. From March 1989 to August 1992, he was with Electronics and Telecommunications Research Institute (ETRI) as a head of the applied radio section, where he was responsible for fundamental research and new technology development for radio direction finding system. In September 1992, he joined the faculty of college of electronics and information at Kyung Hee University, Korea, where he is currently a professor. His research interests include mobile communication systems, spectrum engineering, smart antenna and SDR.

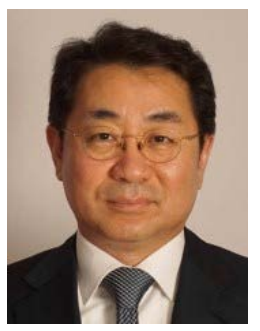

Dr. Bong Gyou Lee who is a professor at Graduate School of Information has served as a Vice President, CISO, CPO and a director of Communications Policy Research Center(CPRC) in Yonsei University. Dr. Lee received a B.A. from the Department of Economics at Yonsei University and M.S, Ph.D. from Cornell University. During 2007 and 2008 he served as Commissioner of the Korea Communications Commission.

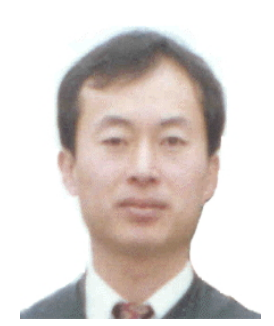

Kyeongmin Song received the M.S. degree in electronic engineering from Soongsil University in 2000. From 1999 to 2010, he was with Onse Telecom Co. as a head of the development section. In 2010, he founded Wibtel Co., LTD where he is currently a president.. His research interests include RF propagation prediction, EMC, RADAR and space weather. 